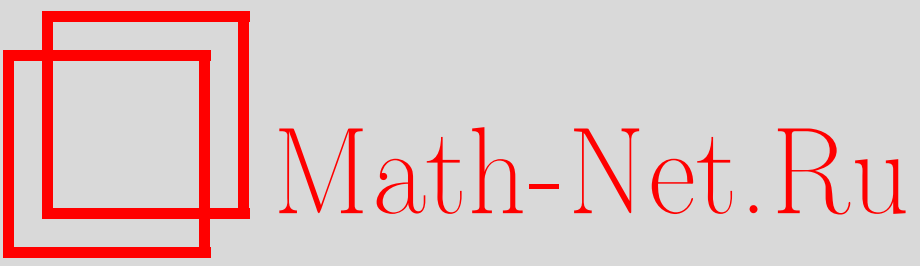

Г. Н. Сергазы, Применение $B$-сплайна в методе эмпирической декомпозиции мод для разложения двумерного временного ряда на внутренние моды, Итоги науки и техн. Сер. Соврем. мат. и ее прил. Темат. обз., 2021, том 194, 163-166

DOI: https://doi.org/10.36535/0233-6723-2021-194-163-166

Использование Общероссийского математического портала Math-Net.Ru подразумевает, что вы прочитали и согласны с пользовательским соглашением

http: //www.mathnet.ru/rus/agreement

Параметры загрузки:

IP: 52.87 .193 .239

26 апреля 2023 г., 12:41:36 


\title{
ПРИМЕНЕНИЕ В-СПЛАЙНА \\ В МЕТОДЕ ЭМПИРИЧЕСКОЙ ДЕКОМПОЗИЦИИ МОД ДЛЯ РАЗЛОЖЕНИЯ ДВУМЕРНОГО ВРЕМЕННОГО РЯДА НА ВНУТРЕННИЕ МОДЫ
}

\author{
(c) 2021 г. $\quad$ Г. Н. СЕРГАЗЫ
}

\begin{abstract}
АннотАция. Идея эмпирической декомпозиции мод (EMD) была предложена Н. Э. Хуаном как итерационный метод декомпозиции нелинейной и нестационарной функции на сумму составляющих, называемых внутренними модами. В первую очередь EMD была представлена как метод анализа одномерных временных рядов. Метод позволил извлекать быстро осциллирующие компоненты сигнала с нулевыми средними, наложенные на медленные колебания. В данной работе описывается алгоритм эмпирической декомпозиции двумерного временного ряда с применением В-сплайна в итерационном процессе EMD.
\end{abstract}

Ключевые слова: эмпирическая декомпозиция мод, двумерный временной ряд, -сплайн, процесс просеивания, дефектоскопия рельсов.

\section{APPLICATION OF $B$-SPLINES \\ WITHIN THE METHOD OF EMPIRICAL MODE DECOMPOSITION FOR EXPANDING A TWO-DIMENSIONAL TIME SERIES INTO INTERNAL MODES}

\author{
(c) 2021 G. N. SERGAZY
}

\begin{abstract}
The idea of empirical mode decomposition (EMD) was proposed by N. E Huang as an iterative method of decomposition of a nonlinear and nonstationary function into the sum of components called internal modes. First, EMD was presented as a method for analyzing one-dimensional time series. The method made it possible to extract rapidly oscillating signal components with zero averages superimposed on slow oscillations. In this paper, we describe an algorithm for empirical decomposition of a two-dimensional time series using a $B$-spline in an EMD iterative process.
\end{abstract}

Keywords and phrases: empirical mode decomposition, two-dimensional time series, $B$-spline, sieving process, rail flaw detection.

AMS Subject Classification: 41A15

1. Введение. Эмпирическая декомпозиция мод (EMD) является адаптивным методом анализа данных. Данный метод не нуждается в априори заданных базисных функциях, чем и отличается от других методов анализа данных, таких как Фурье-анализ и вейвлеты. Также особенностью EMD является его применение к нестационарным и нелинейным данным. Под данными понимается вещественный временной ряд $\left\{s_{t}\right\}_{t=1}^{\infty}$. Временной ряд называется нестационарным, если совместные распределения $\left[s_{t_{1}}, s_{t_{2}}, \ldots, s_{t_{n}}\right]$ и $\left[s_{t_{1}+\tau}, s_{t_{2}+\tau}, \ldots, s_{t_{n}+\tau}\right]$ одинаковы для всех $t_{i}$ 
и $\tau$. Результатом применения эмпирической декомпозиции к некоторому сигналу есть множество функций, которые обладают следующими свойствами:

(i) все локальные максимумы положительны, а локальные минимумы отрицательны;

(ii) количество нулей и количество экстремумов функции отличается не более чем на 1 ;

(iii) средняя линия верхней и нижней огибающих, построенных по локальным максимумам и локальным минимумам, соответственно, является нулевая линия;

(iv) все они ортогональны в пространстве $L_{2}(0, T)$.

Данный метод извлекает из сигнала осцилляторы с нулевой средней линией.

Эмпирическая декомпозиция мод в первую очередь была применена для вещественных временных рядов (см. [3]). В [5,6] были представлены расширенные на двумерный временной ряд методы декомпозиции. Проблемы, с которыми сталкивается оригинальный метод, также встречаются и в расширенном методе. Одной из проблем является выбор подходящей интерполяции для извлечения средней линии (см. [2]). Было решено использовать метод декомпозиции для двумерного случая с применением $B$-сплайна.

Изучение двумерного метода эмпирической декомпозиции обусловлено поиском наилучшего метода анализа данных в развертке типа $A$ при дефектоскопии рельсовых путей двумя пьезоэлектрическими преобразователями.

2. Метод просеивания. EMD не нуждается в априори заданных функциях, они извлекаются непосредственно из самого сигнала. Алгоритм разложения оригинального сигнала на базис, которым и являются внутренние моды, называется методом просеивания. Это итерационный метод, основанный на интерполировании экстремумов и вычитании медиан. Хотя в основном метод применяется к дискретному временному ряду, для наглядности алгоритм будет описан для непрерывной функции $s(t), t \in[0, T]$.

2.1. Оригинальный алгоритм. Согласно авторам EMD [3], алгоритм декомпозиции состоит из следующих шагов:

I: найти локальные максимумы и минимумы $s$;

II: построить верхнюю огибающую $u(t)$ и нижнюю огибающую $l(t)$, интерполируя кубическими сплайнами локальные максимумы и локальные минимумы соответственно;

III: вычислить среднюю линию $m(t)=\frac{1}{2}(u(t)+l(t))$;

IV: вычислить $h(t)=s(t)-m(t)$;

$\mathrm{V}$ : процесс останавливается, если $h$ является внутренней модой. В обратном случае $h$ рассматривается как сигнал, и к нему применяются шаги $\mathrm{I}-\mathrm{V}$.

В вычислительных целях вместо проверки свойств, описанных во Введении, используется один из следующих критериев:

(а) Пусть $h_{k-1}$ и $h_{k}$ были вычислены на $(k-1)$-й и $k$-й итерациях. Определим

$$
S D_{k}:=\frac{\left\|h_{k-1}-h_{k}\right\|_{L_{2}(0, T)}}{\left\|h_{k-1}\right\|_{L_{2}(0, T)}} .
$$

Процесс останавливается, если $S D_{k}$ меньше, чем заранее выбранный порог.

(b) Пусть $N_{k}$ - количество пересечений нуля функцией $h_{k}$, а $E_{k}-$ количество экстремумов. Тогда процесс останавливается, если за $S$ итераций достигается условие $\left|N_{k}-E_{k}\right|<1$, где $S$ заранее выбрано.

Первая внутренняя мода обозначается $c_{1}$, а $r_{1}=s-c_{1}$ есть остаток. Алгоритм повторяется для $r_{1}$, чтобы получить следующую внутреннюю моду. Этот процесс продолжается, пока последний остаток $r_{n}$ не станет монотонной или не будет иметь только один экстремум, без учета граничных точек. Исходный сигнал может быть представлен в виде

$$
s=\sum_{j=1}^{n} c_{j}+r_{n} .
$$


2.2. Применение $B$-сплайна в методе просеивания. Определим $B$-сплайны порядка $k$ для произвольного набора узлов. Для возрастающей последовательности точек $\tau_{j}, j \in \mathbb{Z} j$-й $B$-сплайн порядка $k$ есть

$$
B_{j, k, \tau}(t):=\left(\tau_{j+k}-\tau_{j}\right)\left[\tau_{j}, \ldots, \tau_{j+k}\right](x-t)_{+}^{k-1}, \quad t \in \mathbb{R},
$$

где

$$
(x-t)_{+}^{k-1}= \begin{cases}0, & x<t \\ (x-t)^{k-1}, & x \geqslant t\end{cases}
$$

(см. [1]). Множество $B$-сплайнов образует базис в пространстве сплайнов порядка $k$ с узлами $\tau_{j}$, $j \in \mathbb{Z}$.

Пусть $\tau^{s}:=\left\{\tau_{j}: j \in \mathbb{Z}\right\}$ - множество экстремумов сигнала $s$. Точки $\tau_{j+l}, l=0,1, \ldots, k$, принадлежат носителю $B$-сплайна $B_{j, k, \tau}$, и он строго положителен в узлах $\tau_{j+1}, \ldots, \tau_{j+k-1}$. Для узлов строго внутри носителя $B_{j, k, \tau}$ определен линейный функционал

$$
\lambda_{j, k, \tau^{s}}(s):=\frac{1}{2^{k-2}} \sum_{l=0}^{k-2} C_{k-2}^{l} s\left(\tau_{j+l+1}\right) .
$$

Оператор

$$
V_{\tau^{s}, k}(t):=\sum_{j \in \mathbb{Z}} \lambda_{j, k, \tau^{s}}(s) B_{j, k, \tau}(t)
$$

заменяет среднюю линию верхней и нижней огибающих в оригинальном алгоритме [4]. K примеру, при $k=4$ оператор имеет вид

$$
V_{\tau^{s}, 4}(t)=\sum_{j \in \mathbb{Z}} \frac{1}{4}\left[s\left(\tau_{j+1}\right)+2 s\left(\tau_{j+2}\right)+s\left(\tau_{j+3}\right)\right] B_{j, 4, \tau}(t) .
$$

Тогда алгоритм просеивания состоит из следующих шагов (см. [4]):

I: найти локальные экстремумы сигнала $s$;

II: применить оператор $V_{\tau^{s}, k} \mathrm{~K} s$;

III: вычислить $h=s-V_{\tau^{s}, k}$;

IV: процесс останавливается, если $h$ является внутренней модой. Для проверки используется один из критериев из предыдущего раздела. В обратном случае $h$ рассматривается как сигнал, и к нему применяются шаги I-IV.

3. Алгоритм EMD для двумерного временного ряда. Идея алгоритма эмпирической декомпозиции в двумерном случае заключается в том, что двумерный сигнал является быстро вращающимися сигналами, наложенными на медленные (см. [5]). Суть метода в данном случае заключается в том, чтобы извлечь быстрые вращения как внутренние моды, а самое медленное вращение будет приниматься за тренд. Очевидным способом расширения алгоритма на двумерный случай является использование оригинального метода на каждый временной ряд по отдельности. Но при этом теряется зависимость между двумя компонентами сигнала. Данную проблему было предложено решить с помощью поворотов сигнала вокруг временной оси и применения декомпозиции к модификациям сигнала (см. $[5,6])$.

Пусть $s(t)=\left\{s_{1}(t), s_{2}(t)\right\}, t \in[0, T]$ - двумерный сигнал. Алгоритм эмпирической декомпозиции в двумерном случае с применением $B$-сплайна состоит из следующих шагов:

I: пусть $w_{\theta_{v}}(t)$ - проекция на плоскость $\left(t, s_{1}\right)$ поворота сигнала $s$ на угол $\theta_{v}$, где $\theta_{v}=2 \pi v / V$, $v=1,2, \ldots, V$

II: найти узлы локальных экстремумов $\left\{\tau_{j}^{v}\right\}_{v=1}^{V}$ соответствующих $w_{\theta_{v}}(t)$;

III: применить оператор $V_{\tau^{s}, k}$ к каждой проекции $w_{\theta_{v}}(t)$;

IV: вычислить двумерное среднее $m(t)$ :

$$
m(t) \approx \frac{1}{v} \sum_{v=1}^{V} V_{\tau^{w_{\theta}, k}}(t)
$$

$\mathrm{V}:$ вычислить $h(t)=s(t)-m(t)$; 
VI: проверить, является ли $h$ двумерной внутренней модой. Для этого надо проверить все проекции $h$ при повороте на углы $\theta_{v}=2 \pi v / V, v=1,2, \ldots, V$ по одному из выше описанных критериев. В обратном случае шаги алгоритма повторяются для $h(t)$.

После извлечения первой внутренней моды весь алгоритм повторяется для разности сигнала и моды. Алгоритм останавливается, когда из остатка уже нельзя извлечь моду.

4. Заключение. Для выявления дефектов на рельсовых путях используются развертки типа $A$ и $B$, где $A$-развертка представляет полученный сигнал в форме времени по абсциссе и амплитуды сигнала по ординате, а $B$-развертка есть изображение информативных сигналов в области сечения объекта контроля. Специалисту проще определить дефект на $B$-развертке, так как за счет визуализации данных имеет более информативный формат. Но для машинного обучения дефектоскопии удобнее использовать $A$-развертку, которая является временным рядом. Для выявления дефектов по одному каналу используются два пьезоэлектрических прибора. Данные, полученные из этих приборов, представляют собой двумерный временной ряд. Двумерная эмпирическая декомпозиция мод позволяет произвести предварительную обработку данных, за счет которого можно избавиться от сильных шумов и произвести анализ тренда для прогнозирования о наличии дефекта или о длине уже имеющегося.

\section{СПИСОК ЛИТЕРАТУРЫ}

1. Де Бор К. Практическое руководство по сплайнам. - М.: Радио и связь, 1985.

2. Huang N. E. Introduction to the Hilbert-Huang transform and its related mathematical problems// Interdisc. Math. Sci. - 2005. - 5. - P. 1-26.

3. Huang N. E., Shen Z., Long R. S., Wu M. C., Shih H. H., Zheng Q., Yen N. C., Tung C. C., Liu H. H. The empirical mode decomposition and the Hilbert spectrum for nonlinear and non-stationary time series analysis// Proc. Roy. Soc. London Ser. A. - 1998. — 454. - P. 903-995.

4. Riemenschneider S., Liu B., Xu Y., Huang N. E. B-Spline based empirical mode decomposition// Interdisc. Math. Sci. - 2005. - 5. - P. 27-55.

5. Rilling G., Flandrin P., Goncalves P., Lilly J. M. Bivariate empirical mode decomposition// IEEE Signal Process. Lett. - 2007. - 14, № 2. - P. 936-939.

6. Tanaka T., Mandic D. P. Complex empirical mode decomposition// IEEE Signal Process. Lett. — 2007. 14, № 2. - P. 101-104.

Сергазы Галимжан Нургазыулы

Московский государственный университет им. М. В. Ломоносова

E-mail: gsergazy@cs.msu.su 